„DER AUSTAUSCH IN DER WISSENSCHAFT IST EIN GRUNDPFEILER FÜR ZUKÜNFTIGE LÖSUNGEN VON MENSCHHEITSFRAGEN. DAHER IST DIE ASSOZIATION GROSSBRITANNIENS AN ERASMUS+ UND DAS KOMMENDE FORSCHUNGSFÖRDERUNGSPROGRAMM HORIZON EUROPA ANZUSTREBEN.“

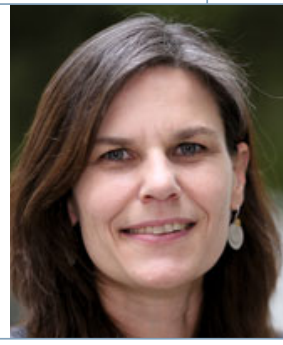

Muriel Helbig

\title{
Bedeutung des Brexits für die europäische Wissenschaftsgemeinschaft
}

DOI: $10.1007 / \mathrm{s} 12268-020-1360-\mathrm{z}$ (C) Die Autorin 2020

Brüssel im November 2017. Ein Saal in Blau-Weiß, festlich gekleidete Menschen aller Altersgruppen: Im Palais des BeauxArts wird das 30. Jubiläum des europäischen Austauschprogramms Erasmus+ begangen. Auf der Bühne werden bewegende persönliche Geschichten erzählt, vor allem aber wird ein großes Bild von einem Programm gezeichnet, das Europa verändert hat, indem es einzelne Menschen verändert hat. Und Erasmus+ ist noch lange nicht alles. Hinter der Vielzahl an Programmen und Stipendien einer gemeinsamen europäischen Bildungsund Wissenschaftspolitik steht ein Grundgedanke: Die Schicksalsfragen der Menschheit lassen sich nur gemeinsam lösen, und der Austausch in der Wissenschaft ist ein Grundpfeiler der zukünftigen Lösungen.

In dieser europäischen Wissenschaftslandschaft spielt Großbritannien eine gewichtige Rolle. Hier finden sich einige der besten Universitäten des Kontinents, hier arbeiten viele exzellente Wissenschaftlerinnen und Wissenschaftler, hierhin zieht es Studierende aus aller Welt. Unter Studierenden, Promovierenden und Forschenden aus Deutschland belegt Großbritannien im Ranking der DAAD-Förderung nach EU-Ländern den zweiten Rang - übertroffen in der Beliebtheitsskala nur von Spanien.

Das Interesse an einer engen internationalen Zusammenarbeit ist ungebrochen hoch. So fordert die britische Wissenschaftsorganisation Royal Society für die Zeit nach dem EU-Ausstritt, hochqualifizierte internationale Wissenschaftlerinnen und Wissenschaftler im Land zu halten sowie den Zugang zu dem EU-Forschungsförderprogramm „Horizon“ oder dem Forschungsinfrastruktur-Konsortium „ERIC“ aufrechtzuerhalten.

Studierendenaustausch steht für viele forschungsorientierte britische Hochschulen allerdings weniger im Fokus als beispielsweise für britische Colleges. Großbritannien nimmt zwar seit vielen Jahren eine große
Zahl internationaler Studierender, Wissenschaftlerinnen und Wissenschaftler auf, den umgekehrten Weg jedoch - von Großbritannien in die Welt - wählen nur wenige. Nach Deutschland beispielsweise kamen 2018 rund 2.000 Briten, während es 15.700 Deutsche auf die Insel zog. Insgesamt 8.000 Auslandsaufenthalte nach und von Großbritannien wurden hierbei vom Deutschen Akademischen Austauschdienst (DAAD) gefördert; 75 Prozent übrigens durch eine Mobilitätsförderung über Erasmus+.

Der Austritt Großbritanniens aus der EU ist daher ein herber Verlust für den europäischen Hochschulraum und die Forschungsfähigkeiten innerhalb der EU. DAAD-Präsident Joybrato Mukherjee formuliert es so: Der Brexit ist ein Schlag gegen die Bündelung der intellektuellen Kapazitäten Europas. Es trifft uns alle, wenn ein so wichtiger Partner wie Großbritannien aus dem gemeinsamen Bündnis ausscheidet.

Zwar läuft zunächst bis Ende 2020 mit Blick auf den akademischen Austausch alles wie bisher, da eine Übergangsfrist gilt. Erasmus+-Projekte der aktuellen Programmgeneration laufen weiter, auch über 2020 hinaus. Auslandsaufenthalte mit Erasmus+ sind sogar bis Ende Mai 2022 gesichert, beim Thema Aufenthaltsgenehmigung müssen sich Studierende allerdings ab 2021 umorientieren. Dennoch ist der Zeitplan für Verhandlungen für einen Anschlussvertrag zwischen der EU und Großbritannien eng. Daher fordern der DAAD und weitere Wissenschaftsorganisationen, Wissenschaft weit oben auf die Prioritätenliste bei den Gesprächen zu setzen.

Ein gangbarer Weg wäre die Assoziation Großbritanniens an Erasmus+ und das kommende Forschungsförderungsprogramm „Horizon Europa“. Anzustreben wären zudem eine erleichterte Einreise und ein erleichterter Aufenthalt für Studium, Forschung und Ausbildung sowie die Beibehaltung des privilegierten Status von Unionsbürgern in Bezug auf die Studiengebühren in Großbritannien. Erasmus+ und „Horizon 2020“ sind bereits heute für die Teilnahme von Drittstaaten offen. Eine Teilassoziierung wäre somit für Großbritannien möglich, ist jedoch an Bedingungen, wie z. B. eine finanzielle Beteiligung, geknüpft.

Auch außerhalb dieser Verhandlungen laufen weitere Aktivitäten. Beispielsweise bemühen sich britische Hochschulen, einen Tochtercampus auf dem Kontinent zu errichten, zudem werden bilaterale Kooperationen mit einzelnen deutschen Hochschulen geschlossen. Kritiker befürchten, dass es hierdurch zu einer verstärkten Elitenbildung kommen wird: Studierende, die es sich leisten können, werden einen Auslandsaufenthalt absolvieren; Hochschulen, die es sich leisten können, eine Forschungskooperation. Auch wenn ein „hard Brexit" vermieden und Lösungen gefunden werden können, gilt für die Zeit ab 2021 weiterhin: Die zentralen Menschheitsfragen lassen sich nur gemeinsam lösen. Eine enge Zusammenarbeit zwischen der EU und Großbritannien ist daher unabdingbar.

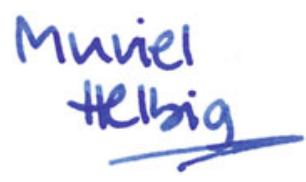

Muriel Helbig, Vizepräsidentin des DAAD und Präsidentin der TH Lübeck

\section{Funding: Open Access funding provided by Projekt DEAL. \\ Open Access: Dieser Artikel wird unter der Creative Commons Namensnennung 4.0 International Lizenz veröffentlicht, welche die Nutzung, Vervielfältigung, Bearbeitung, Verbreitung und Wiedergabe in jeglichem Medium und Format erlaubt, sofern Sie den/die ursprünglichen Autor(en) und die Quelle ordnungsgemäß nennen, einen Link zur Creative Commons Lizenz beifügen un angeben, ob Anderungen vorgenommen wurden. Die in diesem Artikel enthaltenen Bilder und sonstiges Drittmaterial unterliegen ebenfalls der nichts anderes ergibt. Sofern das betreffende Material nicht inter der genannten Creative Commons Lizenz steht und die betreffende Handlung nicht genannten Creative Commons Lizenz steht und die betreffende Handlung nicht Weiterverwendungen des Materials die Einwilligung des jeweiligen Rechteinhabers einzuholen. Weitere Details zur Lizenz entnehmen Sie bitte der Lizenzinformation auf http://creativecommons.org/licenses/by/4.0/deed.de.}

\section{Korrespondenzadresse:}

Dr. Muriel Helbig

Technische Hochschule Lübeck

University of Applied Sciences

Mönkhofer Weg 239

D-23562 Lübeck

praesidentin@th-luebeck.de

www.th-luebeck.de 